\title{
Ear Skin
}

National Cancer Institute

\section{Source}

National Cancer Institute. Ear Skin. NCI Thesaurus. Code C49481.

The skin of the organ responsible for detecting sound and establishing balance. 